\title{
LA SITUACIÓN DE LOS DERECHOS HUMANOS EN EL CONFLICTO DE NAGORNO-KARABAJ. UNA VISIÓN DESDE LOS MECANISMOS DE PROTECCIÓN DE NACIONES UNIDAS
}

\author{
Dorothy Estrada Tanck ${ }^{1}$ \\ Universidad de Murcia
}

\section{Resumen:}

El conflicto entre Armenia y Azerbaiyán sobre el Nagorno-Karabaj presenta hondas raíces históricas y se ha visto exacerbado por el conflicto armado de 2020. En este contexto, a menudo la situación de los derechos humanos de las personas afectadas y, en particular, los de las mujeres y niñas, queda invisibilizada. Así, este artículo analiza el conflicto refiriendo la postura general de la ONU y el Derecho Internacional Público y ampliando el lente, en especial, sobre el Derecho internacional de los derechos humanos y los mecanismos de Naciones Unidas articulados para su protección. Se presenta un enfoque específico sobre los derechos humanos de las mujeres y niñas, dando cuenta de las situaciones empíricas que enfrentan, para estudiarlas después bajo la mirada de los derechos humanos con una perspectiva de género, poniendo énfasis en lo que al respecto han manifestado los principales mecanismos de protección internacional dedicados al tema.

Palabras Clave: Nagorno-Karabaj, ONU, derechos humanos, mecanismos de protección, mujeres y niñas.

Title in English: The human rights situation in the Nagorno-Karabakh conflict: A synopsis from the UN protection mechanisms.

\begin{abstract}
:
The conflict between Armenia and Azerbaijan over Nagorno-Karabakh has deep historical roots and has been exacerbated by the armed conflict of 2020. In this context, the human rights situation of those affected, and especially of women and girls, is often left invisible. Thus, this article analyses the conflict by referring to the general position of the UN and public international law and by widening the lens, in particular, on international human rights law and the UN mechanisms articulated for the protection of those rights. A distinct focus on the human rights of women and girls is presented, giving an account of the empirical situations they face, to then study them through the lens of human rights with a gender perspective, emphasizing what the main international protection mechanisms dedicated to the issue have expressed in this regard.
\end{abstract}

Key words: Nagorno-Karabakh, UN, human rights, protection mechanisms, women and girls

Copyright (C) UNISCI, 2021.

Las opiniones expresadas en estos artículos son propias de sus autores, y no reflejan necesariamente la opinión de UNISCI. The views expressed in these articles are those of the authors, and do not necessarily reflect the views of UNISCI.

\footnotetext{
${ }^{1}$ Dorothy Estrada Tanck es Profesora Contratada Doctora de Derecho Internacional Público y Relaciones Internacionales de la Facultad de Derecho, Universidad de Murcia E-mail:<dorothyestrada@um.es>

DOI: http://dx.doi.org/10.31439/UNISCI-129
} 


\section{Introducción ${ }^{2}$}

Tras años de control ruso y soviético, en 1991 Armenia y Azerbaiyán obtuvieron su independencia. Una libertad costosa que se apoyó en las fronteras soviéticas preexistentes que separaban a los armenios étnicos de Nagorno-Karabaj de su tierra firme. Es uno de los muchos conflictos etnoterritoriales 'congelados' que se libran en los territorios de la antigua Unión Soviética. Antes de la creciente esperanza de unidad entre ambos territorios, la población mayoritariamente armenia de Nagorno-Karabaj (o el 'Alto Karabaj' o 'Artsaj') anunció sus intenciones de separarse de Azerbaiyán. La lucha armada comenzó el 6 de enero de 1992, cuando la región declaró su independencia. Esta primera lucha terminó en 1994, dejando 30.000 víctimas y desplazando a casi un millón de personas. ${ }^{3}$ Los vencedores, los armenios, obtuvieron la independencia de facto y Armenia ocupó el territorio fronterizo de Azerbaiyán. Los perdedores, de Azerbaiyán, tuvieron que desplazar a su población azerí y sufrir que sus territorios estuvieran en manos de los armenios. Aun así, como es bien sabido, en las cuatro resoluciones de 1993 del Consejo de Seguridad de Naciones Unidas (ONU/NU) sobre la ocupación de Armenia a Nagorno Karabaj, la ONU la acepta como territorio de Azerbaiyán y ningún país del mundo reconoce la administración armenia que rige la región, aunque de facto tales resoluciones del CSONU no se hayan implementado hasta hoy en día. ${ }^{4}$

Avanzamos rápidamente hasta 2020, cuando se produce la peor escalada de violencia desde la llamada Guerra de los Cuatro Días, en abril de 2016, que dejó dos centenares de muertos. Hay que situarse, pues, en 2020, cuando Azerbaiyán y su población azerí se habían preparado durante mucho tiempo para un reajuste de cuentas. La capacidad e inversión militar de Azerbaiyán se cuadruplicó y recibió drones y asistencia militar de Turquía. Precisamente con estos drones, en septiembre de 2020, Azerbaiyán atacó la capital de Nagorno-Karabaj, Stepanakert. Las autoridades de Nagorno-Karabaj afirman que casi 1.200 miembros de sus fuerzas de defensa han muerto en los combates, y que también han muerto o resultado heridas personas civiles. Azerbaiyán no ha hecho públicas sus cifras de bajas militares, pero ha dicho que más de 80 civiles han muerto en los combates, incluidos 21 personas en un ataque con misiles contra la ciudad de Barda. ${ }^{5}$ Otras cifras de informes gubernamentales y no gubernamentales indican que durante las hostilidades militares en Nagorno-Karabaj y sus alrededores, entre septiembre y noviembre de 2020, murieron más de 150 civiles. $^{6}$

En este contexto, desde la reanudación de las hostilidades el 27 de septiembre de 2020 y diversos intentos de alto el fuego, incluyendo el acuerdo de paz firmado en noviembre de 2020, distintos mecanismos de protección internacional de derechos humanos han expresado su preocupación sobre la grave situación de los derechos humanos en el terreno. Más

\footnotetext{
${ }^{2}$ Este artículo es resultado de la estancia 21185/EE/19 financiada por la Fundación Séneca-Agencia de Ciencia y Tecnología de la Región de Murcia con cargo al Programa Regional de Movilidad, Colaboración e Intercambio de Conocimiento "Jiménez de la Espada".

${ }^{3}$ Schoch, Bruno (et al.), 'Introduction', en Babayev, Azer, Bruno Schoch y Hans-Joachim Spanger(2020): The Nagorno-Karabakh deadlock, Wiesbaden, Springer, p. 2.

${ }^{4}$ Rehimov, Ruslan, 'Las cuatro resoluciones del Consejo de Seguridad de ONU sobre ocupación de Armenia al Alto Karabaj', Agencia Anadolu, 29 de septiembre de 2020, en https://www.aa.com.tr/es/mundo/las-cuatroresoluciones-del-consejo-de-seguridad-de-onu-sobre-ocupación-de-armenia-al-alto-karabaj-/1990003

5 'Armenia, Azerbaiyán y Rusia firman un acuerdo de paz para Nagorno-Karabaj', BBC News, 10 de noviembre de 2020, en https://www.bbc.com/mundo/noticias-internacional-54886462

${ }^{6}$ Véase, e.g., Human Rights Watch, 'Submission to the Committee on the Elimination of Discrimination against Women. Review of Armenia', 81st Pre-Session, 24 de junio de 2021, en https://www.hrw.org/news/2021/06/24/submission-committee-elimination-discrimination-against-womenreview-armenia
} 
concretamente, el Grupo de Trabajo de la ONU sobre el Uso de Mercenarios ha recibido reiteradas denuncias de ataques deliberados e indiscriminados en zonas pobladas de la zona de conflicto de Nagorno-Karabaj y sus alrededores, así como en otras zonas alejadas de los combates, lo que ha provocado un número creciente de víctimas civiles, incluidas niñas y niños. También se ha informado ampliamente de daños considerables en propiedades e infraestructuras civiles, como escuelas y hospitales, así como de incidentes con periodistas. La organización no gubernamental (ONG) Human Rights Watch, de las pocas ONGs que desde finales de los ochentas ya se pronunciaba sobre las atrocidades cometidas en la región, ${ }^{7}$ también investigó si las fuerzas armadas cumplían con sus obligaciones en virtud del derecho internacional humanitario, y documentó múltiples violaciones por parte de ambos bandos, así como las consecuencias del conflicto para los niños y niñas en edad escolar, en un contexto educativo ya de por sí difícil por el previo cierre escolar a inicios del 2020 derivado del contexto del COVID-19. ${ }^{8}$

Particular atención merece la condición de las mujeres y niñas en el conflicto, condición que con frecuencia queda invisibilizada en las perspectivas tradicionales de análisis de conflictos. Para examinar la situación de los derechos humanos de las mujeres en la región, debe destacarse que el Cáucaso alberga sociedades patriarcales, donde el papel de la mujer está estrictamente definido y controlado por el entorno masculino. Y en tiempos de crisis, diferentes tipos de presiones, como esforzarse por la supervivencia física, cuidar de los niños y niñas y de las personas con discapacidad durante la crisis y el hambre, participar en los combates paralelamente a hacer frente a la violencia de género, los estigmas, los estereotipos y el acoso sexual, recaen generalmente sobre la espalda de las mujeres. ${ }^{9}$ Además, estas sociedades del sur del Cáucaso, gobernadas por hombres, están plagadas de un fuerte nacionalismo. Armenia y Azerbaiyán, tras independizarse de la Unión Soviética, se embarcaron en una fuerte retórica masculina, nacionalista y religiosa que socavó la igualdad de género y el lugar de las mujeres en la sociedad. ${ }^{10}$ Estos factores son clave en la escalada del conflicto, pero también en las razones por las que las mujeres han sido víctimas desiguales de un conflicto que no provocaron y de una paz en la que no pueden participar.

Así, este artículo analiza en el apartado 2 el conflicto desde el Derecho Internacional Público y la postura del Consejo de Seguridad y otros actores y, en particular, del Derecho internacional de los derechos humanos desde el lente de los mecanismos de Naciones Unidas articulados para su protección. Con ese telón de fondo, se pone un enfoque particular sobre la situación de los derechos humanos de las mujeres en el conflicto, a menudo ocultada tras el examen mayoritariamente geopolítico de la crisis. Así, se da cuenta primero en el apartado 3. de las situaciones empíricas que enfrentan las mujeres y niñas, tanto azeríes como armenias, especialmente en el periodo comprendido entre 1991 y 2020 , para estudiar esas situaciones en el apartado 4 bajo la mirada de los derechos humanos de las mujeres y las niñas con una perspectiva de género. Se pone especial énfasis en lo que al respecto han manifestado los principales mecanismos sobre el tema, a saber, el Comité de Naciones Unidas sobre la Eliminación de Todas las Formas de Discriminación contra la Mujer, la Relatora Especial sobre la Violencia contra la Mujer y el Grupo de Trabajo de Naciones Unidas sobre la Discriminación contra las Mujeres y las Niñas. En el apartado 5 se presentan algunas reflexiones finales en torno a la utilidad, retos y aportaciones del sistema internacional

\footnotetext{
${ }^{7}$ Priego Moreno, Alberto, 'Nagorno-Karabag ¿disputa territorial o energética?', UNISCI Discussion Papers, nº2 (2003), p. 9, recuperado a partir de https://revistas.ucm.es/index.php/UNIS/article/view/UNIS0303230018A

${ }^{8}$ Human Rights Watch, op. cit.

9 Tskhvariashvili, Sophio, 'Gender Implications of the Nagorno-Karabakh Conflict: Alternative Feminist Perspectives', Tesis de Máster, Ivane Javakhishvili Tbilisi State University, 2018, p. 8.

${ }^{10}$ Ibid., p. 9.
} 
de derechos humanos.

\section{Postura sobre el conflicto desde la ONU y los mecanismos internacionales de protección de derechos humanos}

\subsection{El conflicto y la paz y seguridad internacionales: la ONU y el Derecho internacional público general}

Como se mencionaba antes, y a reserva de analizar más a fondo la postura de los mecanismos internacionales de protección de los derechos humanos, el Grupo de Trabajo de NU sobre el Uso de Mercenarios expresó que había informes generalizados de que el gobierno de Azerbaiyán, con la ayuda de Turquía, recurría a combatientes sirios para reforzar y mantener sus operaciones militares en la zona de conflicto de Nagorno-Karabaj, incluso en la línea del frente. Según los expertos de la ONU, los combatientes parecían estar motivados principalmente por el beneficio privado, dada la grave situación económica de la República Árabe Siria. Asimismo, el Grupo de Trabajo ha expresado inquietud por el hecho de que los combatientes sirios desplegados en Azerbaiyán estén supuestamente afiliados a grupos armados e individuos que, en algunos casos, han sido acusados de crímenes de guerra y de graves abusos contra los derechos humanos durante el conflicto en Siria, perpetuando así, al parecer, un ciclo de impunidad y arriesgándose a que se produzcan nuevos abusos del derecho internacional. ${ }^{11}$

Cabe a este respecto recordar la prohibición del uso unilateral de la fuerza armada en las relaciones internacionales, contenida en el artículo 2.4 de la Carta de Naciones Unidas, prohibición sobre la que se contempla la estricta excepción de la legítima defensa y 'solo hasta' la intervención de Naciones Unidas (artículo 51 de la Carta de Naciones Unidas).

En ese sentido, y atendiendo a lo señalado por el Grupo de Trabajo de Naciones Unidas sobre Mercenarios, debe enfatizarse la confirmación de esta prohibición del Derecho internacional por la Declaración de Principios anexa a la resolución 2625 (XXV) de la Asamblea General, adoptada en 1970. En esta resolución, el concepto de fuerza armada se comprende en una acepción amplia, incluyendo tanto los ataques armados 'directos' llevados a cabo por las fuerzas regulares de un Estado, como los 'indirectos', es decir, los cometidos por grupos armados enviados o controlados por un Estado que no forman parte de su ejército regular, como en este contexto parecería ser el caso sobre todo de Azerbaiyán y Turquía, según la información revelada.

Como se ha referido, las resoluciones $822,853,874$ y 884 del Consejo de Seguridad de la ONU, de 1993, ${ }^{12}$ constituyen la base fundamental jurídicamente vinculante para una solución política de Nagorno-Karabaj, aunque suscitan interpretaciones contradictorias. Los azerbaiyanos tienden a destacar las frases que afirman la integridad territorial sin reconocer la necesidad de una solución política de Nagorno-Karabaj. Los armenios tienden a utilizar las resoluciones para negar la participación de los militares de la República de Armenia. ${ }^{13}$

Aun así, esas resoluciones del Consejo de Seguridad de la ONU coinciden con dos de

\footnotetext{
${ }^{11}$ Oficina del Alto Comisionado de Naciones Unidas para los Derechos Humanos, 'Mercenaries in and around the Nagorno-Karabakh conflict zone must be withdrawn - UN experts', Comunicado de prensa, 11 November 2020, en https://www.ohchr.org/EN/NewsEvents/Pages/DisplayNews.aspx?NewsID=26494

${ }^{12}$ Resolución 822 (1993), adoptada por el Consejo de Seguridad en su 3205a sesión, el 30 de abril de 1993; Resolución 853 (1993), adoptada por el Consejo de Seguridad en su 3259ª sesión, el 29 de julio de 1993; Resolución 874 (1993), adoptada por el Consejo de Seguridad en su 3292a sesión, el 14 de octubre de 1993; y Resolución 884 (1993), adoptada por el Consejo de Seguridad en su 3313ª sesión, el 12 de noviembre de 1993.

${ }^{13}$ Véase en este sentido el análisis de Jan de Graaf, Wouter, 'What UN Security Council Resolutions Say about Nagorno-Karabakh, and What They Don't Say', Providence, 4 November 2020, en https://providencemag.com/2020/11/united-nations-security-council-resolutions-nagorno-karabakh-armeniaazerbaijan-artsakh/
} 
la Asamblea General de la ONU, así como de muchas organizaciones internacionales, que han exigido el retiro de las fuerzas de ocupación de estos territorios. Ya en 1992, para encontrar una solución pacífica al conflicto, se formó el Grupo de Minsk de la Organización para la Seguridad y la Cooperación en Europa (OSCE), copresidido por Francia, Rusia y Estados Unidos, ${ }^{14}$ pero como puede verse de los hechos posteriores, este grupo fracasó en su objetivo.

En la situación reciente, es de hacerse notar que el Consejo de Seguridad de la ONU, órgano naturalmente llamado a pronunciarse sobre la situación de conflicto armado en la región, con base en su mandato de mantenimiento de la paz y la seguridad internacionales contenido en el Capítulo VII de la Carta de NU, hasta la fecha de escribir el presente artículo, no ha emitido una resolución formal al respecto. Aunque el Consejo se reunió el 19 de octubre de 2020 con la secretaria general adjunta de Asuntos Políticos y de Consolidación de la Paz de la ONU, Rosemary Di Carlo, en sesión cerrada para consultas sobre el conflicto en NagornoKarabaj, no emitió después una resolución y simplemente se ha limitado mediante su portavoz, a apoyar la postura del Secretario General de la ONU, Antonio Guterres, del cese al fuego y la protección de las personas civiles. ${ }^{15}$

Puede aventurarse que esa ausencia del Consejo de Seguridad se basa principalmente en el interés de Rusia -miembro permanente del Consejo de Seguridad y, por tanto, indispensable para la adopción de una resolución-, de mostrar su dominio regional y su control sobre el territorio anteriormente bajo su poder, y constituirse en líder geopolítico y tomador de decisiones a nivel individual, al margen del multilateralismo propio de NU. Con ello, además, refuerza su posición frente a Turquía como adalid regional, y a la vez como mediador eficaz, postura que se ve confirmada si se toma en cuenta el impulso de Moscú del acuerdo de paz firmado precisamente entre Armenia, Azerbaiyán y Rusia, ${ }^{16}$ y apoyado por el Grupo Minsk de la OSCE. Aun con las incógnitas que despierta el acuerdo, se ha considerado que "[d]espués de más de 25 años de absoluta inamovilidad hacia la búsqueda de una solución factible, la firma de este acuerdo de paz ha conseguido desbloquear el eterno estancamiento que impedía avanzar en alguna dirección". ${ }^{17}$

Como parte de su intervención en el proceso de paz, Moscú ha enviado también una misión de paz sobre el terreno y puede estar esperando hasta que su misión esté firmemente establecida y las líneas de contacto definidas. De acuerdo con algunos datos, cuando Rusia propuso que el Consejo de Seguridad apoyara el alto el fuego del 9 de noviembre, Francia y Estados Unidos insistieron en esperar, en busca de más información. En cualquier caso, los funcionarios rusos dicen que el consentimiento de Armenia, junto con el de Azerbaiyán, es suficiente para validar su presencia militar y civil, y añaden que su primera prioridad es

\footnotetext{
${ }^{14}$ Rehimov, Ruslan, op. cit.

${ }^{15}$ Security Council Report, 'Nagorno-Karabakh Consultations', 19 October 2020, en

https://www.securitycouncilreport.org/whatsinblue/2020/10/nagorno-karabakh-consultations.php y Secretaría General de NU, 'Statement attributable to the Spokesperson for the Secretary-General - on Nagorno-Karabakh', 4 December 2020, en https://www.un.org/sg/en/content/sg/statement/2020-12-04/statement-attributable-thespokesperson-for-the-secretary-general-nagorno-karabakh

${ }^{16}$ Véase a este respecto el análisis crítico sobre el tipo particular de ‘internacionalismo’ de Rusia y su llamado a la aplicación de reglas universales, cuando Rusia mantiene, al menos, una posición engañosa sobre el derecho de autodeterminación, y el autor plantea la sugerente pregunta: “¿preceden los intereses materiales rusos al derecho de autodeterminación que reclaman algunos en Tatarstán, Chechenia, Nagorno-Karabaj, Moldavia y Georgia?”, Van Severen, Sebastiaan 'Lavrov's Lament: A Russian take on the rules-based global order', en EJIL:Talk! Blog of the European Journal of International Law, July 2021, en https://www.ejiltalk.org/lavrovs-lament-a-russiantake-on-the-rules-based-global-order/ Véase también Ismailov, Famil: 'Nagorno Karabaj: cómo Rusia ayudó al acuerdo en el conflicto entre Armenia y Azerbayán y asumió "control total" sobre el terreno', en BBC News Rusia, 11 de noviembre de 2020, en https://www.bbc.com/mundo/noticias-internacional-54903249

${ }^{17}$ Setién, Sara, 'Conflicto de Nagorno Karabaj 2020: ¿nos encontramos ante la solución definitiva?', Instituto Español de Estudios Estratégicos, Documentos de Opinión, 54/2020, 1 de diciembre de 2020, p. 20.
} 


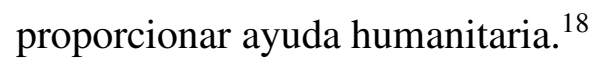

\subsection{El conflicto y los derechos humanos: el Derecho internacional de los derechos humanos, la ONU y sus mecanismos de protección}

En vista de la escalada reciente del conflicto y los sucesos de los últimos años, distintos expertos de los mecanismos de protección internacional de derechos humanos de Naciones Unidas se han pronunciado al respecto. Más allá de esos pronunciamientos específicamente dirigidos al conflicto, existen una serie de instrumentos y estándares aplicables a la situación de forma general, en virtud de la adhesión por parte de Armenia y de Azerbaiyán de los diferentes tratados internacionales de derechos humanos. Conviene, por tanto, proporcionar una breve descripción de estos mecanismos de protección de derechos humanos y su funcionamiento.

$\mathrm{Al}$ respecto, la sociedad internacional en el marco de Naciones Unidas ha creado desde su creación en 1945, distintos órganos para el sistema de promoción, protección y garantía de los derechos humanos, con base en los principios generales de igualdad y no-discriminación. Esos principios fueron reconocidos tanto en la Carta de NU como en la Declaración Universal de Derechos Humanos (DUDH), adoptada por la Asamblea General de la ONU el 10 de diciembre de 1948. Los dos tratados centrales adoptados posteriormente en 1966, el Pacto Internacional de Derechos Civiles y Políticos y el Pacto Internacional de Derechos Económicos, Sociales y Culturales, conforman junto con la DUDH, la llamada 'Carta Internacional de Derechos Humanos', por agrupar a los instrumentos que contemplan los derechos humanos en su globalidad.

Sin embargo, es de hacer notar que, desde la DUDH de 1948 y los dos Pactos de 1966, los Estados han adoptado varios tratados más en materia de derechos humanos, concretamente, en total nueve tratados internacionales (incluyendo a los dos Pactos) y nueve Protocolos Facultativos sobre una amplia gama de temas de derechos humanos

Existen, pues, dos grandes ramas del sistema de derechos humanos, a saber, por un lado, los 'órganos de tratados', creados en virtud de cada uno de los nueve tratados principales de derechos humanos ${ }^{19}$ para su interpretación y la supervisión de su cumplimiento, y por otro, los 'Procedimientos Especiales' del Consejo de Derechos Humanos (también llamados 'órganos de la Carta'), que encuentran su fundamento jurídico directo en la Carta de Naciones Unidas, tratado internacional obligatorio para sus 193 Estados Miembros. Uno de los nueve tratados de derechos humanos referidos es la Convención sobre la Eliminación de Todas las Formas de Discriminación Contra la Mujer (CEDAW, por sus siglas en inglés), de la cual tanto Armenia como Azerbaiyán son Estados Parte.

Una gran mayoría de Estados son actualmente parte en todos o en varios de los tratados internacionales de derechos humanos. Como es bien sabido, cuando un Estado ratifica un tratado internacional de derechos humanos, contrae la obligación jurídica de implementar las

\footnotetext{
18 'Improving Prospects for Peace after the Nagorno-Karabakh War', International Crisis Group, Crisis Group Europe Briefing no 91, Baku/Yerevan/Tbilisi/Istanbul/Moscow/Brussels, 22 December 2020, p. 15.

${ }^{19}$ Resulta importante considerar el resto de los nueve tratados internacionales de derechos humanos, además de los dos Pactos: la Convención internacional sobre la eliminación de todas las formas de discriminación racial (adoptada el 21 de diciembre de 1965), la Convención sobre la eliminación de todas las formas de discriminación contra la mujer (de 18 de diciembre de 1979) -particularmente importante para el tema del presente estudio-, la Convención contra la tortura y otros tratos o pena crueles, inhumanos o degradantes (de 10 de diciembre de 1984), la Convención sobre los derechos del niño (de 20 de noviembre de 1989); la Convención internacional sobre la protección de los derechos de todos los trabajadores migratorios y de sus familiares (de 18 de diciembre de 1990); la Convención sobre los derechos de las personas con discapacidad (de 13 de diciembre de 2006); y la Convención internacional para la protección de todas las personas contra las desapariciones forzadas (de 20 de diciembre de 2006). Puede, por tanto, entenderse que todos estos tratados internacionales también constituyen, junto con los instrumentos referidos anteriormente, la 'Carta Internacional de los Derechos Humanos' del siglo XXI.
} 
disposiciones establecidas en ese tratado y observar sus normas. También acepta presentar informes periódicamente al comité correspondiente sobre los avances en la implementación del tratado en cuestión. Para cada tratado hay un Comité -el 'órgano del tratado'-, que revisa estos informes y monitorea qué tan exitosamente los Estados están implementado los derechos allí contenidos.

Los órganos creados en virtud de los tratados, los Comités de Tratados, están compuestos por expertos y expertas independientes y se reúnen para examinar los informes periódicos de los Estados Parte sobre su cumplimiento del tratado respectivo. También pueden publicar Comentarios o Recomendaciones Generales sobre temas de derechos humanos relacionados con los tratados que supervisan. Los distintos Comités de Tratados tienden a seguir modelos similares en la documentación. Estos comentarios generales se publican en los 'Instrumentos Internacionales de Derechos Humanos: Recopilación de las observaciones generales y recomendaciones generales adoptadas por órganos creados en virtud de tratados de derechos humanos'.20

De forma paralela y complementaria, surge el sistema de Procedimientos Especiales del Consejo de Derechos Humanos, órgano inter-estatal subsidiario de la Asamblea General de NU. Estos procedimientos, que pueden ser Relatores Especiales, Expertos Independientes o Grupos de Trabajo, tienen facultades de realizar visitas oficiales a los Estados, analizar situaciones de derechos humanos y realizar recomendaciones tanto a los Estados Parte de tratados de derechos humanos, como a los que no lo son. Es decir, su cobertura geográfica es global, y con ello ayudan a llenar los 'huecos' del sistema de Órganos de tratados. Asimismo, su cobertura temática es amplia también, pues si bien su mandato se refiere a una dimensión específica en materia de derechos humanos, en su labor los Procedimientos Especiales pueden hacer uso de toda la gama de tratados internacionales de derechos humanos, así como de la interpretación que de éstos han realizado los Comités de tratados -una fuente que se clasificaría dentro de los instrumentos llamados de soft law-, y también pueden emplear las otras fuentes del Derecho Internacional Público en la materia, por ejemplo, la costumbre internacional, los principios generales del derecho, la jurisprudencia y la doctrina. ${ }^{21}$

Concretamente sobre el conflicto en cuestión, los Procedimientos Especiales de NU, especialmente el Grupo de Trabajo de Naciones Unidas sobre el Uso de Mercenarios, han señalado que los mercenarios en la zona de conflicto de Nagorno-Karabaj y sus alrededores deben ser retirados. Más concretamente, los expertos en derechos humanos de NU tomaron nota del acuerdo alcanzado el 9 de noviembre de 2020 para poner fin a las hostilidades en el conflicto de Nagorno-Karabaj, y manifestaron su esperanza de que dicho acuerdo condujera a una paz duradera en la región. No obstante, estaban preocupados por el uso de mercenarios en la zona de conflicto y sus alrededores hasta que se alcanzó este acuerdo, y expresaron su alarma por las consecuencias devastadoras para la población civil, con pocas perspectivas de rendición de cuentas. $^{22}$

Como ya se adelantaba, el Grupo de Trabajo de NU sobre el Uso de Mercenarios ha señalado en noviembre de 2020 que los combatientes sirios involucrados en el conflicto en apoyo a Azerbaiyán parecían estar motivados esencialmente por necesidad económica, debido a las precarias condiciones en Siria. Al parecer, a sus familiares se les prometió una

\footnotetext{
${ }^{20}$ Documento HRI/GEN/1/Rev.9, Vol. I y Vol. II.

${ }^{21}$ Sobre fuentes, véase el artículo que constituyó un parteaguas en su momento respecto de los entendimientos menos tradicionales del Derecho Internacional Público, y con una perspectiva feminista: Charlesworth, Hilary, Christine Chinkin y Shelley Wright, 'Feminist Approaches to International Law', en The American Journal of International Law, vol. 85, n⿳. 4 (October 1991), pp. 613-645.

${ }^{2}$ Oficina del Alto Comisionado de Naciones Unidas para los Derechos Humanos, 'Mercenaries in and around the Nagorno-Karabakh conflict zone must be withdrawn - UN experts', op. cit.
} 
compensación económica y la nacionalidad turca en caso de muerte de los combatientes. El modo en que se reclutó, transportó y utilizó a estas personas en la zona de conflicto de NagornoKarabaj y sus alrededores parecía ajustarse a la definición de mercenario, tal y como se establece en los instrumentos jurídicos internacionales pertinentes, incluida la Convención Internacional contra el Reclutamiento, la Utilización, la Financiación y el Entrenamiento de Mercenarios, de la que Azerbaiyán es parte. Además, señaló que los informes indican que Turquía participó en el reclutamiento y traslado a gran escala de hombres sirios a Azerbaiyán a través de facciones armadas, algunas de las cuales están afiliadas al Ejército Nacional Sirio. El Grupo de Trabajo indicó que también había recibido informes que dan cuenta de que Armenia participó en el despliegue de ciudadanos extranjeros para luchar en el conflicto de NagornoKarabaj. ${ }^{23}$

Por otro lado, varios Procedimientos Especiales -el Relator Especial sobre la tortura y otros tratos o penas crueles, inhumanos o degradantes; el Grupo de Trabajo sobre Desapariciones Forzadas o Involuntarias, y la Relatora Especial sobre ejecuciones extrajudiciales, sumarias o arbitrarias-, realizaron conjuntamente un llamamiento en febrero de 2021, por el que pidieron la pronta liberación de los prisioneros de guerra y otros cautivos del conflicto de Nagorno-Karabaj, así como la devolución de los cadáveres a las familias para su entierro con el debido respeto a las costumbres culturales. Enfatizaron que todas las personas privadas de libertad por motivos relacionados con el conflicto deben ser devueltas a sus hogares, y los familiares de los asesinados deben poder recibir los restos mortales de sus seres queridos, en consonancia con el acuerdo de alto el fuego firmado el 9 de noviembre de $2020 .^{24}$

Expresaron su alarma por las denuncias sobre violaciones de derechos humanos contra civiles y militares cautivos, y sobre la profanación de los muertos, así como por los informes de que los prisioneros de guerra y otras personas protegidas han sido objeto de ejecuciones extrajudiciales, desapariciones forzadas, torturas y otros malos tratos. Subrayaron que tales actos, cuando se perpetran en un conflicto armado, pueden constituir también crímenes de guerra. $^{25}$

Por ello, hicieron un llamamiento a las autoridades de Armenia y Azerbaiyán para que llevaran a cabo investigaciones exhaustivas, rápidas, independientes e imparciales sobre las denuncias de graves violaciones de los derechos humanos cometidas durante el conflicto y sus secuelas, con el fin de que los autores rindan cuentas y se ofrezca reparación a las víctimas. Enfatizaron que estas acciones facilitarán la verdad, la reconciliación y la curación. ${ }^{26}$

Puede observarse así cómo los mecanismos de derechos humanos de Naciones Unidas sirven para visibilizar situaciones no sólo desde la perspectiva política, sino también y, sobre todo, desde el lente de los derechos humanos y reafirmar y recordar a los Estados las soluciones que el Derecho internacional de los derechos humanos indica que deberían adoptarse como parte del cumplimiento de sus obligaciones en la materia. En este marco, especial atención merece la situación de los derechos humanos de las mujeres y las niñas, que a menudo queda negada, minimizada o invisibilizada en los análisis geopolíticos, e incluso en los exámenes generales sobre distintas violaciones de derechos humanos.

\footnotetext{
${ }^{23}$ Ibid.

${ }^{24}$ Oficina del Alto Comisionado de Naciones Unidas para los derechos Humanos, 'Nagorno-Karabakh: captives must be released - UN experts', Comunicado de prensa, 1 de febrero de 2021, en https://www.ohchr.org/EN/NewsEvents/Pages/DisplayNews.aspx ?NewsID=26702\&LangID=E ${ }^{25}$ Ibidem.

${ }^{26}$ Ibidem.
} 


\section{La situación particular de los derechos humanos de las mujeres y las niñas}

\subsection{Las mujeres y niñas azeríes}

El largo conflicto abordado ha tenido graves consecuencias en Azerbaiyán, y las mujeres azeríes se han visto especialmente afectadas en distintos tipos de condiciones vivenciales que, a su vez, implican frecuentemente también violaciones a derechos humanos, las más frecuentes siendo los desplazamientos forzados y la situación de refugio, las asesinatos y pérdida masiva de la vida, las agresiones sexuales, y la pérdida de identidad cultural. ${ }^{27}$

En 2020, tras los acuerdos conseguidos por el Gobierno de Azerbaiyán, se prometió devolver a los azeríes expulsados una gran parte del territorio del sur de Azerbaiyán. Sin embargo, 29 años después del conflicto original, son pocos los que quedan para poder hacerlo, y son varios los que siguen teniendo sus tierras bajo control armenio. En ese contexto, empezando por el desplazamiento forzado y la condición de personas refugiadas, cabe referir que cientos de miles de mujeres azeríes se vieron obligadas a abandonar sus hogares y ahora viven como refugiadas o como desplazadas internas. Los azeríes son la minoría musulmana de Azerbaiyán en Nagorno-Karabaj, reacia a la mayoría de los referendos y movimientos de unidad dirigidos en la región por armenios mayoritariamente cristianos. Las mujeres azeríes constituyen el setenta y uno por ciento de los azeríes desplazados por la fuerza y, tras casi veinte años de desplazamiento, un gran número de ellas sigue viviendo en el umbral de la pobreza. En 2012, 600.000 azeríes vivían en condiciones de desplazamiento 'temporal', y se calcula que 400.000 siguen viviendo hacinados en viviendas 'temporales' de mala calidad, como escuelas reconvertidas, dormitorios y edificios abandonados, mientras que unos 120.000 se han trasladado en los últimos años de los 'campamentos de tiendas de campaña' para desplazados internos/refugiados y otras viviendas 'temporales' similares a asentamientos construidos por el gobierno. ${ }^{28}$

Muchas mujeres azeríes desplazadas/refugiadas tenían un alto nivel de formación y ocupaban puestos importantes como médicas, académicas, profesoras, líderes de cooperativas y administradoras en sus comunidades de origen antes del desplazamiento. Y antes, las mujeres, o bien sólo podían encontrar un empleo de menor nivel o se encontraban en situación de desempleo, mientras que otras obtenían ingresos a través del trabajo en la economía informal. Además, por la ideología patriarcal que durante mucho tiempo ha sido un elemento destacado en la cultura azerí hacia las mujeres, las muertes y desapariciones de hombres condenaron a muchas mujeres a mayores daños sociales, psicológicos y sociales. Del mismo modo, en algunos casos, las personas desplazadas internas/refugiadas eran vistas como de estatus inferior o como 'diferentes' en comparación con otros azeríes, incluso tratándose de mujeres que se refieren a otras mujeres desplazadas/refugiadas de forma despectiva, con lo que se comunica implícitamente que un 'refugiado' es indeseablemente diferente de otros azeríes. ${ }^{29}$

Para continuar con la siguiente situación trágica enfrentada por las mujeres azeríes, hay que referirse a los asesinatos. Al respecto, las mujeres azeríes no quedaron excluidas de los numerosos asesinatos del conflicto. Los días 25 y 26 de febrero de 1992, los armenios -con

\footnotetext{
${ }^{27}$ Este apartado forma parte de una investigación más amplia sobre la situación de los derechos humanos de las mujeres y niñas, por parte del Grupo de Trabajo de Naciones Unidas sobre la Discriminación contra las Mujeres y las Niñas, del que la autora es miembro.

${ }^{28}$ Mehrangiz, Najafizadeh, 'Ethnic Conflict and Forced Displacement: Narratives of Azeri IDP and Refugee Women from the Nagorno-Karabakh War', en Journal of International Women's Studies, vol. 14, n 1 (2013), p. 168.

${ }^{29}$ Ibidem.
} 
la ayuda del $366^{\circ}$ Regimiento de Infantería Mecanizada del ejército ruso- atacaron brutalmente la comunidad de Khojaly, en Nagorno-Karabaj, poblada por unos 7.000 azeríes, y mataron a 613 azeríes, entre ellos 106 mujeres, 83 niños y 79 ancianos azeríes. ${ }^{30}$ Esta masacre se tituló 'Masacre de Khojaly' (o Masacre de Jóyali) y marcó a Azerbaiyán, que la consideró una limpieza étnica y un genocidio. ${ }^{31}$

Asimismo, la violación y los delitos sexuales, un horror traumático común de la guerra, no están exentos de las duras realidades del conflicto. En el conflicto de Nagorno-Karabaj, la violación, como arma de guerra específica del género, se utilizó con extrema frecuencia contra los 'enemigos' para humillarlos y vengarse. Y la violación, como arma, se utilizó exclusivamente contra las personas del sexo femenino, por abusar del significado simbólico que tienen para las naciones. Este tema es altamente estigmatizado y ampliamente instrumentalizado para la movilización nacional. La directora del Centro de Mujeres, Paz y Seguridad, en Azerbaiyán, declaró "Sí, las mujeres fueron violadas... [...] Sólo para demostrar que se humilla al adversario. Hubo muchas mujeres que fueron violadas, pero para nosotros es un tema cerrado, es un tema tabú, puede haber muy pocos que lo admitan. Prácticamente nadie habla de este tema". ${ }^{32}$ Lo anterior revela que, en la cultura azerí, la violación es una herramienta extremadamente dañina que excluye a las mujeres de cualquier inclusión social si se habla de ella, lo que suele provocar el silencio de las víctimas.

A pesar de que es el tema más tabú y menos investigado por todas las partes del conflicto, Sophio Tskhvariashvili descubrió en su investigación 'Implicaciones de género del conflicto de Nagorno-Karabaj', que la mayoría de los encuestados confirmó que hubo una violencia brutal y acosos sexuales contra las mujeres, desde el comienzo del conflicto. Por poner un ejemplo ilustrativo, Arzu, una mujer de 52 años que participó en el proceso de intercambio de rehenes y prisioneros de guerra, confirmaba: "Sí, la captura de las mujeres siempre terminaba con una violación". ${ }^{33}$ Esta actitud brutal, especialmente hacia las mujeres, ha provocado numerosos suicidios por dignidad -cuando las mujeres se suicidaban para no ser capturadas por 'el enemigo' - y asesinatos por dignidad -cuando las mujeres eran asesinadas para que no fueran tomadas como rehenes-. Y este tipo de suicidios y asesinatos se convirtieron incluso en parte de las narrativas nacionalistas heroicas de la guerra. Según Gulnara Mehdiyeva, una de las principales representantes del movimiento feminista en Azerbaiyán, en la mentalidad caucásica, preservar la dignidad y proteger a 'tus mujeres' representa el valor central de las naciones y en el contexto militar las mujeres asumen principalmente el papel de víctimas o rehenes, que se sacrifican heroicamente para proteger su dignidad, más que de héroes que participan en acciones militares. ${ }^{34}$

Las defensoras y defensores de derechos humanos y feministas de Azerbaiyán que han criticado la guerra y han pedido que la gente se preocupara por los civiles que estaban en peligro por los combates, han recibido mensajes de odio, algunos vulgares y otros llamándolas 'traidoras'. Estos se intensificaron después de que los armenios atacaran objetivos civiles en las ciudades azerbaiyanas de Ganja y Barda. Ahora que la guerra ha terminado, Mehdiyeva quiere reorientar su activismo para abordar el modo en que las mujeres se han visto afectadas. Señala

\footnotetext{
${ }^{30}$ Ibid., p. 165.

${ }^{31}$ Ibidem. Véase también Jofre Leal, Pablo, 'Jodyali: La necesidad de verdad y justicia', Blog, Telesurtv.net, 21 de febrero de 2020, en https://www.telesurtv.net/bloggers/Jodyali-La-necesidad-de-verdad-y-justicia-202002210001.html y Boletín IEEE número 12, Octubre/diciembre 2018 - Instituto Español de Estudios Estratégicos, p. 14.

${ }^{32}$ Tskhvariashvili, Sophio, op. cit., p. 41.

${ }^{33}$ Ibid., p. 40.

${ }^{34}$ Véase la referencia a Gulnara Mehdiyeva, en Samadov, Bahruz, 'Perspectives: Azerbaijan's peace activists face harassment, and a reckoning. For those of us advocating for progressive values in Azerbaijan, the war has been a setback', Eurasianet, 20 November 2020, en https://eurasianet.org/perspectives-azerbaijans-peace-activists-faceharassment-and-a-
} 
que las imágenes de las mujeres se instrumentalizaron en la guerra, por ejemplo, identificando la 'patria' con un cuerpo de mujer que debe ser protegido, mientras que los problemas de la mujer real han sido ignorados. La activista por los derechos humanos de las mujeres revela que "La guerra ha planteado nuevas cuestiones: las experiencias de las mujeres que son madres de soldados, enfermeras, mujeres en el ejército; las experiencias de las esposas de los soldados con estrés postraumático, las mujeres refugiadas, etc.”.35

En este apartado sobre las condiciones medulares vividas por las mujeres, debe destacarse también que los desplazamientos y las matanzas también han dejado una oscura y profunda herida en la identidad y la cultura de los azari de Nagorno-Karabaj. Distintos testimonios dan cuenta de la resiliencia y la esperanza de volver a sus tierras, y expresan estas emociones y sentimientos tanto verbalmente como por escrito, sobre todo a través de la narración y la poesía, por ejemplo, a través del famoso poema 'Si pudiera volver a ver Kelbajar'. ${ }^{36}$ Esta identidad y la esperanza en el futuro son fundamentales tanto para que las mujeres afronten las experiencias del desplazamiento como para su empoderamiento.

\subsection{Las mujeres y niñas armenias}

En la sociedad armenia, las mujeres en varios entornos son vistas como aparatos para hacer soldados. Por ejemplo, en las reuniones sociales se hace un singular brindis por la anfitriona de la familia por criar buenos hijos y buenas futuras madres de los soldados. Esta cultura se repite en la región, ya que se transmite de generación en generación, y quienes no apoyan o siguen esta cultura suelen ser considerados antiarmenios. ${ }^{37}$ Varias activistas feministas tienen una opinión muy crítica sobre este tema. ${ }^{38}$ Consideran que esta actitud hacia las mujeres está muy extendida y es problemática, ya que alimenta y reproduce también otras opresiones estructurales. Señalan que las crisis bélicas cambiaron totalmente el tejido social y convirtieron a las mujeres únicamente en madres y a los hombres en héroes. Eso, como violencia directa, provoca también otras formas de violencia estructural. En muchas ocasiones se ve a las mujeres sólo como la presencia necesaria para dar a luz y no participar en ninguna toma de decisiones, ni en la vida social. Concluyen que "las mujeres son obligadas a convertirse en meras fábricas de producción de soldados, son sólo cuerpos, no son percibidas como seres humanos". 39

Ante la toxicidad de la narrativa masculina/guerra/género, las mujeres armenias de Nagorno-Karabaj sufren una violencia masculina repetitiva y legitimada. Un ejemplo perfecto de ello son los soldados varones que regresan del conflicto. Vuelven con un comportamiento muy salvaje y violento, sobre todo, cuando van armados. Hay recuentos de que todos los que tienen acceso a las armas querían lucirse en la plaza. Podían coger la ametralladora y lucirse e incluso matarse públicamente cuando había peleas. ${ }^{40}$ Los hombres que volvían de la guerra se consideraban los héroes de la misma y se sentían más masculinos, bajo esta concepción dominante de masculinidad. El director del Centro de Mujeres, Paz y Seguridad descubrió que "cuando ellos (los hombres) volvieron a sus familias, cambiaron fisiológicamente, sintieron la necesidad de tomar la posición dominante. Y las estadísticas muestran que la violencia en la familia en ese momento aumentó mucho". La violencia familiar es una tendencia principal en la nación, de tal manera que los divorcios

\footnotetext{
35 Ibid.

${ }^{36}$ Mehrangiz, Najafizadeh, op. cit., p. 177.

${ }^{37}$ Sarade, Manisha, 'Gendered dimensions of the Nagorno-Karabakh conflict', LSE Blogs, Actualizado el 18 de noviembre de 2020, en https://blogs.lse.ac.uk/wps/2020/11/18/gendered- dimensions-of-the-nagorno-karabakhconflict/

38 Tskhvariashvili, Sophio, op. cit., p. 39.

${ }^{39}$ Ibidem.

${ }^{40}$ Ibid., p. 45.
} 
han aumentado drásticamente para las mujeres que buscan valientemente el fin de la violencia. El Ministerio de Justicia armenio descubrió que el $76 \%$ de las mujeres que se divorcian lo hacen cuando son económicamente independientes de sus maridos, lo que demuestra la también dura represión cultural existente. ${ }^{41}$ E incluso en el caso del divorcio, el sistema político y judicial está demasiado influenciado por esta cultura represiva. Como ejemplo ilustrativo, cabe considerar el caso de un hombre que estuvo golpeando a su mujer durante diecisiete años y también a su hija, de las formas más humillantes, increíbles e inhumanas. La esposa huyó de él y encontró refugio en el Centro de Acogida de Mujeres. Hubo un juicio y finalmente su pena fue sólo una multa, equivalente a 100 dólares - 50000 dramas. Y la justificación fue que "bueno, merece mucho respeto por ser miembro de la Unión de Veteranos, no puede ser encarcelado - luchó en la guerra". ${ }^{42}$

Aunque la 'cultura de la guerra heroica' armenia ha perjudicado mucho a las mujeres, debe señalarse que también les ha ofrecido una plataforma para implicarse en el conflicto, hecho que no comparten las mujeres azeríes que no combaten. Las mujeres de Armenia y Karabaj han tenido la posibilidad de luchar o asistir a los combates en el frente. El Consejo de Coordinación 'Mujeres por Karabaj' adoptó una resolución el 6 de diciembre de 2000 durante la mesa redonda 'Las mujeres dicen 'No' a un mundo derrotista'. En noviembre de 2001 se creó un Consejo de Coordinación en el marco de 'La Organización para la Liberación de Karabaj', en el que las participantes no ocultaron su actitud radical ante el problema de Karabaj, que, en su opinión, debía resolverse por la fuerza. En la mesa redonda, las mujeres propusieron la formación de un batallón femenino, y también exigieron al gobierno un informe sobre los gastos del ejército nacional. ${ }^{43}$ Desde entonces, las mujeres sirven en el lado armenio del ejército de Karabaj. Una mujer, que servía en el ejército de Karabaj como señalizadora, reprodujo la profética frase: "En la guerra no hay mujeres; ¡todos son hombres! Comíamos con los hombres, dormíamos en la misma habitación y sobrevivíamos juntos a la muerte de amigos y compañeros de armas. Se estableció una amistad especial entre nosotros. Una relación superior a la que existe entre un hombre y una mujer". ${ }^{44}$ Las mujeres del ejército armenio de Karabaj han sido señaladas como caídas en combate, y también hubo un gran aumento de alistamientos en la guerra de 2020.

En materia de desplazamiento forzado, se calcula que al menos el 50\% de la población se ha visto obligada a huir entre bombardeos y combates. Toda esta población es de etnia armenia y puebla la región de Nagorno-Karabaj. El defensor de los derechos en Karabaj, Artak Beglaryan, declaró: "Según nuestras estimaciones preliminares, alrededor del 50\% de la población de Karabaj y el $90 \%$ de las mujeres y los niños - entre 70.000 y 75.000 personas - han sido desplazados". ${ }^{45}$

Por lo que toca a los asesinatos, a partir de la madrugada del 27 de septiembre de 2020, Azerbaiyán comenzó a lanzar ataques aéreos indiscriminados (incluidos ataques con vehículos aéreos no tripulados) y ataques de artillería contra la República de Artsaj (República de Nagorno-Karabaj) a lo largo de toda la línea de la frontera. Estos ataques y los siguientes asaltos militares causaron la muerte de muchos civiles. De las 72 víctimas civiles,

\footnotetext{
${ }^{41}$ Ibid., p. 40.

${ }^{42}$ Ibid., p. 46.

${ }^{43}$ Shahnazarian, Nona, 'A Good Soldier and a Good Mother: New Conditions and New Roles in the NagornoKarabakh War', The Journal of Power Institutions in Post-Soviet Studies,(2017), en https://journals.openedition.org/pipss/4241

${ }^{44}$ Ibidem.

${ }^{45}$ Véase 'Half of Nagorno-Karabakh's population displaced by fighting', Deutsche Welle, 7 October 2020, en https://www.dw.com/en/half-of-nagorno-karabakhs-population-displaced- by-fighting/a-55183758
} 
12 eran mujeres. ${ }^{46}$ Azerbaiyán, por su parte, se ha negado a entregar datos y se niega a reconocer estos asesinatos.

\section{Un cúmulo de sufrimientos: respuesta de los mecanismos de protección de derechos humanos de las mujeres y niñas}

En resumen, puede decirse que, desde la independencia hasta los numerosos conflictos recurrentes en la región, no se ha encontrado ninguna respuesta a la seguridad y los derechos de las mujeres y niñas azeríes y armenias. Las mujeres de ambos grupos étnicos sufren enormemente las normas y los conflictos. Según los datos recogidos en la investigación para un informe del Banco Mundial (basado en Azerbaiyán y Georgia), menos del 9\% de los encuestados declararon que querrían una niña si tuvieran un solo hijo. ${ }^{47}$ La política patriarcal y las relaciones de poder que prevalecen en Armenia y Azerbaiyán no son nada novedosas, sin embargo, las circunstancias militarizadas afectan a las relaciones de poder de género en diferentes aspectos de la vida social y política, y podemos observar que la explotación de las mujeres en estos contextos crece de diferentes formas tanto en el ámbito privado como en la típica familia caucásica, donde los roles de género se distribuyen tradicionalmente entre el marido sustentador y el ama de casa cuidadora. ${ }^{48}$

Incluso cuando ambas culturas y grupos se unen, surgen estas preguntas de división y subyugación. En las familias mixtas armenio-azerbaiyanas -que pudieron permanecer juntas en una región alterando los apellidos y ocultando sus identidades-, si una mujer no "reproduce" a los miembros de la nación se le priva de un sentido de identidad y estima nacional, no se le considera patriótica. Por lo tanto, la función reproductora de las mujeres hace avanzar el discurso del 'pueblo en el poder', que percibe la expansión de la población como el interés primordial de las naciones. ${ }^{49} \mathrm{El}$ papel de la mujer como portadora y reproductora de la nación en términos naturales y culturales puede considerarse, sin duda, como una parte central de la narrativa del proyecto nacional dominante en el Cáucaso. Es interesante observar los paradigmas del yo/los otros en el caso de las familias mixtas armenio-azerbaiyanas, que consiguieron permanecer juntas en un condado cambiando los apellidos y ocultando sus identidades. En estos casos, si una mujer no es 'reproductora' de los miembros de la nación, pueden perder el valor nacional e incluso la identidad nacional: "En lo que respecta a las mujeres, no son 'portadoras de la nación' y no son objeto de la nacionalidad. Así, por un lado, no es una mujer armenia, si es madre de hijos azeríes, esposa de azeríes, abuela de azeríes, etc. [...]. [A las mujeres casadas con azeríes] se las llama 'zorras', 'comerciantes', 'no armenias'.50

La práctica de la violencia, el acoso sexual y la violación fue utilizada por todos los bandos del conflicto y los autores justificaban sus acciones como venganza y respuesta al mismo comportamiento del bando contrario. Incluso se percibía como la forma de derrotar al enemigo y fuente de orgullo. Ahora las mujeres han empezado a hablar de las violaciones que han sufrido, y además de la actitud de sus maridos y hermanos que admitían con orgullo haber violado a azerbaiyanos o armenios, porque se consideraba que era algo bueno que cada hombre o cada soldado debía hacer.

Al respecto, ningún tribunal o resolución internacional ha venido a tratar la condición de los derechos humanos en la región de Nagorno-Karabaj de forma integral. Sin embargo, varios mecanismos internacionales de protección de derechos humanos, cada

\footnotetext{
${ }^{46}$ Defensor del Pueblo de la República de Artsaj, 'Informe Provisional sobre los casos de asesinatos de civiles en Artsaj por parte de las Fuerzas Armadas de Azerbaiyán', 2021, en https://artsakhombuds.am/en/document

${ }^{47}$ Sarade, Manisha, op. cit.

48 Tskhvariashvili, Sophio, op. cit., p. 39.

${ }^{49}$ Sarade, Manisha, op. cit.

${ }^{50}$ Tskhvariashvili, Sophio, op. cit., p. 38.
} 
uno actuando en ejercicio de sus competencias, y en ocasiones de forma coordinada, han intentado dar respuesta a las numerosas y complejas violaciones de derechos humanos derivadas del conflicto, incluyendo en lo que toca a aquéllas en contra de las mujeres y niñas y las que les afectan de forma particular o desproporcionada. Se examinan aquí algunos de los principales instrumentos en este sentido, tanto por parte de los Órganos de tratados como por parte de los Procedimientos Especiales del Consejo de Derechos Humanos.

En cuanto a los Órganos de tratados, explicados antes en el apartado 2 de este artículo, el Comité de la Convención sobre la Eliminación de Todas las Formas de Discriminación contra la Mujer (Comité CEDAW), es el órgano central por revisar.

Cabe enfatizar que ya desde 1979 la Convención sobre la eliminación de todas las formas de discriminación contra la mujer (CEDAW, por sus siglas en inglés), de la cual, como se ha referido, son Estados Partes tanto Armenia como Azerbaiyán, consagraba el principio de la igualdad entre mujeres y hombres (artículo 2,a.) y definía que discriminación contra la mujer es "toda distinción, exclusión o restricción basada en el sexo que tenga por objeto o resultado menoscabar o anular el reconocimiento, goce o ejercicio por la mujer, independientemente de su estado civil, sobre la base de la igualdad del hombre y la mujer, de los derechos humanos y las libertades fundamentales en las esferas política, económica, social, cultural y civil o en cualquier otra esfera" (artículo 1).

Así pues, el Comité CEDAW ha revisado diversos informes periódicos tanto de Armenia como de Azerbaiján. Desde la fecha del conflicto más reciente de las seis semanas de lucha armada en 2020, al Comité le ha tocado examinar sólo el informe de Armenia (las últimas Conclusiones Finales del Comité sobre Azerbaiyán son de 2015 y su revisión siguiente será después de 2021). Respecto de la situación en Armenia, el Comité ha realizado en 2021 varias solicitudes al Estado, desde las que se refieren a puntos generales y transversales como la igualdad de género y las causas subyacentes y estructurales de la discriminación contra las mujeres, hasta las que se refieren más concretamente al contexto del conflicto armado.

El Comité CEDAW ha especificado que, a la luz de las recomendaciones anteriores del Comité (CEDAW/C/ARM/CO/5-6, párr. 9) de que se acelere la aprobación de una ley independiente sobre la no discriminación, en consonancia con los artículos 1 y 2 b) de la Convención, que incluya una definición exhaustiva de la discriminación contra la mujer, de conformidad con la Recomendación General No. 28 del Comité (2010), Armenia debe aclarar la situación actual de su proyecto de ley 'Sobre la garantía de la igualdad jurídica' y el plazo para su aprobación. En el informe se indica que el artículo 29 de la Constitución prohíbe la discriminación y que el proyecto de ley, preparado por el Ministerio de Justicia, define la discriminación directa e indirecta. El estado había afirmado que, debido a la pandemia del COVID-19, el Servicio de Estadística interrumpió la recopilación de información sobre la discriminación (CEDAW/C/ARM/7, párrafos 15 a 18), por lo que el Comité CEDAW solicita que Armenia se sirva indicar cuándo se reanudará la recopilación de datos desglosados. ${ }^{51}$

Sobre el contexto del conflicto armado, le ha pedido a Armenia describir las medidas adoptadas para atender las necesidades de las mujeres afectadas por el conflicto armado en cuanto al acceso a la educación, la salud y las oportunidades económicas, en consonancia con la Recomendación General No. 30 del Comité sobre las mujeres en la

\footnotetext{
${ }^{51}$ Comité CEDAW, 'Lista de cuestiones y preguntas en relación con el séptimo informe periódico de Armenia', CEDAW/C/ARM/Q/7, julio de 2021, párrs. 1 y 2.
} 
prevención de conflictos, en situaciones de conflicto y posteriores a los conflictos (2013). ${ }^{52}$

Asimismo, y en un cruce con la Agenda de Mujeres, Paz y Seguridad del Consejo de Seguridad de la ONU, le ha solicitado a Armenia que indique las medidas adoptadas para intensificar los esfuerzos encaminados a la resolución de conflictos, de conformidad con las resoluciones del Consejo de Seguridad 1820 (2008), 1888 (2009), 1889 (2009), 1960 (2010), 2106 (2013) y 2122 (2013), y hacer participar plenamente a las mujeres en todas las etapas del proceso de paz. El Comité CEDAW le ha pedido en particular a Armenia, que se sirva proporcionar al Comité información actualizada sobre el estado de los planes para ultimar y aprobar el plan de acción nacional para aplicar la resolución 1325 (2000) del Consejo de Seguridad sobre la mujer, la paz y la seguridad, en cooperación con las organizaciones de mujeres, teniendo en cuenta todo el espectro del programa del Consejo sobre la mujer y la paz y la seguridad reflejado en las resoluciones del Consejo mencionadas. ${ }^{53}$

En cuanto a los Procedimientos Especiales, los dos principales procedimientos enfocados específicamente en temas de violación a derechos humanos de las mujeres y niñas, a saber, la Relatora Especial sobre la Violencia contra la Mujer, sus Causas y Consecuencias, y el Grupo de Trabajo sobre la Discriminación contra las Mujeres y las Niñas, han articulado diversos estándares de protección que ofrecen una guía sobre cómo aplicar las disposiciones concretas de los instrumentos jurídicos de derechos humanos a la situación particular del conflicto.

La Relatora Especial sobre la Violencia contra la Mujer, sus Causas y Consecuencias, realizó una visita oficial a Azerbaiyán en 2014. Como resultado de esa visita, emitió un informe en el que realiza varias observaciones y recomendaciones al Estado.

La Relatora notó cómo las mujeres desplazadas internas y refugiadas que tuvieron que huir de sus hogares como consecuencia del conflicto de la región de Nagorno-Karabaj registraron una mayor tasa de prevalencia de la violencia de pareja (26\%) en comparación con las encuestadas que nunca fueron objeto de desplazamiento forzado. También destacó que varios datos demuestran que la prevalencia de la violencia de pareja entre las mujeres desplazadas internas y refugiadas es un $7 \%$ mayor que en otros entornos. ${ }^{54}$

En su apartado general de observaciones, la Relatora Especial reconoció el compromiso del Estado con la promoción y protección de los derechos humanos y la adopción de medidas legales específicas para lograr la igualdad de género y la no discriminación. Sin embargo, expresó su preocupación por la falta o la limitada aplicación de las leyes relativas a todas las formas de violencia contra la mujer. La Relatora Especial, en línea con lo que se ha expuesto en el apartado 3 del presente artículo, observó que la violencia contra la mujer estaba ampliamente aceptada en la sociedad y, en cierta medida, por las propias mujeres, debido a los valores patriarcales tradicionales arraigados en la sociedad, lo que perpetuaba aún más la impunidad.

Concretamente, la Relatora Especial notaba con preocupación que [A]unque la mayoría de los entrevistados reconocieron que la violencia contra la mujer estaba muy extendida,...el alcance real del fenómeno era muy difícil de evaluar debido, entre otras cosas, a la falta de información fiable, a que no se denuncian todos los casos, a que se da

\footnotetext{
52 Ibid., párr. 22.

${ }^{53}$ Ibidem.

${ }^{54}$ Informe de la Relatora Especial sobre la violencia contra la mujer, sus causas y consecuencias, Rashida Manjoo, Adición, Misión a Azerbaiyán, A/HRC/26/38/Add.3, 18 de junio de 2014, párr. 8.
} 
prioridad a la mediación y a la reconciliación en los asuntos relacionados con las violaciones de los derechos de la mujer y a la escasa aplicación de las leyes que abordan la cuestión de la rendición de cuentas. La responsabilidad del Estado de actuar con la debida diligencia para eliminar la violencia contra las mujeres es una obligación en virtud del derecho internacional de los derechos humanos a la que el Gobierno se ha comprometido. ${ }^{55}$

Al respecto, la Relatora Especial realizó varias recomendaciones concretas a Azerbaiyán, entre ellas, el establecimiento de servicios gratuitos, sostenibles y apoyados por el Estado para las mujeres víctimas de la violencia, incluyendo una línea de ayuda gratuita que funcione las 24 horas del día y los siete días de la semana, refugios de emergencia y centros de apoyo, asistencia jurídica y médica, apoyo psicosocial y medidas de rehabilitación accesibles a todas las mujeres del país, sin discriminación.

Asimismo, le recomendó introducir medidas especiales de carácter temporal para lograr la igualdad sustantiva en los ámbitos en los que las mujeres están infrarrepresentadas y desfavorecidas, mediante la introducción, por ejemplo, de cuotas para fomentar una mayor participación de las mujeres en la vida política y en los puestos de decisión del Gobierno; introducir medidas especiales de carácter temporal para fomentar el avance de las mujeres en el empleo; y garantizar un apoyo sostenible a las actividades generadoras de ingresos para las mujeres, incluidas las marginadas, en particular las desplazadas internas y las mujeres con discapacidad. ${ }^{56}$

Por su parte, el Grupo de Trabajo sobre la Discriminación contra las Mujeres y las Niñas, en relación con las situaciones vividas por las mujeres azeríes y armenias referidas en el apartado 3 anterior, especialmente si pensamos en la situación de las defensoras de derechos humanos y activistas feministas en Armenia y Azerbaiyán, ha aportado varios estándares generales que sirven de hoja de ruta a la gestión del conflicto y a la reconstrucción de ambas sociedades en la región de Nagorno-Karabaj con perspectiva de derechos humanos y de género.

Por señalar el más relevante a ese respecto, cabe destacar que el Grupo de Trabajo en uno de sus informes (A/HRC/23/50), ha señalado que la estigmatización, el acoso y los ataques directos se utilizan para silenciar y desacreditar a las mujeres líderes, trabajadoras comunitarias, defensoras de los derechos humanos y mujeres políticas, siendo ellas a menudo objetos de violencia de género, como el abuso verbal basado en su sexo; y pudiendo experimentar por su labor intimidación, ataques y también ser asesinadas. El Grupo de Trabajo ha enfatizado que esas situaciones incumplen el deber de los Estados, bajo la CEDAW y oros tratados aplicables, como el Pacto Internacional de Derechos Civiles y Políticos, de garantizar la participación de las mujeres en la vida pública y de ejercer la debida diligencia para prevenir y reparar este tipo de violaciones, incluyendo aquellas que vienen de actores privados.

Es de desear que el propio Grupo de Trabajo, así como otros mecanismos, tanto órganos de tratados como Procedimientos Especiales, por ejemplo, el Comité sobre los Derechos del Niño- puedan pronunciarse o seguir revisando con mayor profundidad la situación de los derechos humanos de las mujeres y niñas en la región, visibilizar las violaciones que sufren y recomendar caminos de solución a adoptarse por los Estados y el resto de actores involucrados, con base en el Derecho internacional de los derechos humanos.

\footnotetext{
${ }^{55}$ Ibid., párr. 81.

${ }^{56}$ Ibid., párr. 84, h), i) y j).
} 


\section{Reflexiones finales}

Evaluar y proteger los derechos humanos, y en especial, los de las mujeres y las niñas en una región en la que todavía hay un conflicto en curso que está blindado por Estados extranjeros, se vuelve especialmente complejo.

En ese contexto, los mecanismos de derechos humanos de la ONU han tomado una postura más decidida que el órgano clásico del derecho internacional público con vocación para el mantenimiento de la paz y seguridad internacionales, a saber, el Consejo de Seguridad. Ello ha sido así en parte debido a la postura de Rusia de constituirse en el líder regional y mediador entre Armenia y Azerbaiyán en el conflicto, países otrora bajo su poder político.

Las mujeres de la región de Nagorno-Karabaj, tanto azeríes como armenias y en diferentes grados, sufren violencia, marginación, falta de libertad económica, sexismo, violaciones y muy poca o ninguna participación en los resultados de la guerra y la paz. En el ámbito nacional, sólo algunos organismos han realizado estudios y denuncias, como el Defensor de los Derechos Humanos de la República de Artsaj o las organizaciones activistas feministas, pero su impacto se quedado limitado con el entorno social patriarcal que se ha visto exacerbado con el conflicto bélico y el resurgimiento abierto de nacionalismos.

Al respecto, tanto los órganos de tratados de derechos humanos como el Comité CEDAW, y los Procedimientos Especiales, como la Relatora Especial sobre la Violencia contra la Mujer, sus Causas y Consecuencias, y el Grupo de Trabajo sobre la Discriminación contra las Mujeres y las Niñas, han articulado diversos estándares de protección que ofrecen una guía y una hoja de ruta sobre cómo aplicar las disposiciones concretas de los instrumentos jurídicos de derechos humanos a la situación particular del conflicto.

Se revela así cómo los mecanismos de protección internacional de derechos humanos de Naciones Unidas sirven para visibilizar situaciones que en ocasiones se vuelven difíciles de enfrentar en el entorno nacional, y para dar luz a esas condiciones, no sólo desde la perspectiva política, sino también y, sobre todo, desde el lente de los derechos humanos. Con ello, los mecanismos de derechos humanos reafirman y recuerdan a los Estados las medidas que el Derecho internacional de los derechos humanos indica que deberían adoptarse como parte del cumplimiento de sus obligaciones en la materia, a fin de encarnar la promesa de los derechos humanos universales de todas las personas del Nagorno-Karabaj y, en particular, de sus mujeres y niñas, que aún esperan la igualdad, la justicia y una paz duradera. 


\section{Bibliografía}

'Armenia, Azerbaiyán y Rusia firman un acuerdo de paz para Nagorno-Karabaj', BBC News, 10 de noviembre de 2020, en https://www.bbc.com/mundo/noticias-internacional-54886462

Charlesworth, Hilary, Christine Chinkin y Shelley Wright, 'Feminist Approaches to International Law', en The American Journal of International Law, vol. 85, nº 4 (October 1991), pp. 613-645.

Comité de Naciones Unidas sobre la Eliminación de Todas las Formas de Discriminación contra la Mujer, 'Lista de cuestiones y preguntas en relación con el séptimo informe periódico de Armenia', CEDAW/C/ARM/Q/7, julio de 2021.

Consejo de Seguridad de Naciones Unidas, Resolución 822 (1993), adoptada por el Consejo de Seguridad en su 3205ª sesión, el 30 de abril de 1993.

, Resolución 853 (1993), adoptada por el Consejo de Seguridad en su 3259a sesión, el 29 de julio de 1993.

, Resolución 874 (1993), adoptada por el Consejo de Seguridad en su 3292a sesión, el 14 de octubre de 1993.

, Resolución 884 (1993), adoptada por el Consejo de Seguridad en su 3313 sesión, el 12 de noviembre de 1993.

, Security Council Report, 'Nagorno-Karabakh Consultations', 19 de octubre de 2020, en https://www.securitycouncilreport.org/whatsinblue/2020/10/nagorno-karabakhconsultations.php

Defensor del Pueblo de la República de Artsaj, 'Informe Provisional sobre los casos de asesinatos de civiles en Artsaj por parte de las Fuerzas Armadas de Azerbaiyán', 2021, en https://artsakhombuds.am/en/document

'Half of Nagorno-Karabakh's population displaced by fighting', Deutsche Welle, 7 de octubre de 2020, en https://www.dw.com/en/half-of-nagorno-karabakhs-population-displaced- byfighting/a-55183758

Human Rights Watch, 'Submission to the Committee on the Elimination of Discrimination against Women. Review of Armenia', 81st Pre-Session, 24 June 2021, en https://www.hrw.org/news/2021/06/24/submission-committee-elimination-discriminationagainst-women-review-armenia

'Improving Prospects for Peace after the Nagorno-Karabakh War', International Crisis Group, Crisis Group Europe Briefing $\mathrm{n}^{\mathbf{0}}$ 91, Baku/Yerevan/Tbilisi/Istanbul/Moscow/Brussels, 22 December 2020.

Instituto Español de Estudios Estratégicos, Boletín IEEE número 12, octubre/diciembre 2018.

Ismailov, Famil, 'Nagorno Karabaj: cómo Rusia ayudó al acuerdo en el conflicto entre Armenia y Azerbayán y asumió "control total" sobre el terreno', en BBC News Rusia, 11 November 2020, en https://www.bbc.com/mundo/noticias-internacional-54903249

Jan de Graaf, Wouter, 'What UN Security Council Resolutions Say about Nagorno-Karabakh, and What They Don't Say', Providence, 4 November 2020, en https://providencemag.com/2020/11/united-nations-security-council-resolutions-nagornokarabakh-armenia-azerbaijan-artsakh/

Jofre Leal, Pablo, 'Jodyali: La necesidad de verdad y justicia', Blog, Telesurtv.net, 21 de febrero de 2020, en https://www.telesurtv.net/bloggers/Jodyali-La-necesidad-de-verdad-y- 
justicia-20200221-0001.html

Mehrangiz, Najafizadeh, 'Ethnic Conflict and Forced Displacement: Narratives of Azeri IDP and Refugee Women from the Nagorno-Karabakh War', en Journal of International Women's Studies, vol. 14, n 1, (2013), pp. 161-183.

Oficina del Alto Comisionado de Naciones Unidas para los Derechos Humanos, 'Mercenaries in and around the Nagorno-Karabakh conflict zone must be withdrawn - UN experts', Comunicado de prensa, 11 de noviembre de 2020, en

https://www.ohchr.org/EN/NewsEvents/Pages/DisplayNews.aspx?NewsID=26494

'Instrumentos Internacionales de Derechos Humanos: Recopilación de las observaciones generales y recomendaciones generales adoptadas por órganos creados en virtud de tratados de derechos humanos', Documento HRI/GEN/1/Rev.9, Vol. I y Vol. II.

'Nagorno-Karabakh: captives must be released - UN experts', Comunicado de prensa, 1 de febrero de 2021, en https://www.ohchr.org/EN/NewsEvents/Pages/DisplayNews.aspx?NewsID=26702\&LangID= $\mathrm{E}$

Priego Moreno, Alberto, 'Nagorno-Karabag ¿disputa territorial o energética?', UNISCI Discussion Papers, n² (2003), en

https://revistas.ucm.es/index.php/UNIS/article/view/UNIS0303230018A

Rehimov, Ruslan, 'Las cuatro resoluciones del Consejo de Seguridad de la ONU sobre ocupación de Armenia al Alto Karabaj', Agencia Anadolu, 29 de septiembre de 2020, en https://www.aa.com.tr/es/mundo/las-cuatro-resoluciones-del-consejo-de-seguridad-de-onusobre-ocupación-de-armenia-al-alto-karabaj-/1990003

Relatora Especial de Naciones Unidas sobre la Violencia contra la Mujer, sus Causas y Consecuencias, Rashida Manjoo, Informe al Consejo de Derechos Humanos, Adición, Misión a Azerbaiyán, A/HRC/26/38/Add.3, 18 de junio de 2014.

Samadov, Bahruz, 'Perspectives: Azerbaijan's peace activists face harassment, and a reckoning. For those of us advocating for progressive values in Azerbaijan, the war has been a setback', Eurasianet, 20 de noviembre de 2020, en https://eurasianet.org/perspectivesazerbaijans-peace-activists-face-harassment-and-a-

Sarade, Manisha, 'Gendered dimensions of the Nagorno-Karabakh conflict', LSE Blogs, Actualizado el 18 November 2020, en https://blogs.lse.ac.uk/wps/2020/11/18/gendereddimensions-of-the-nagorno-karabakh-conflict/

Schoch, Bruno (et al.), 'Introduction', en Babayev, Azer, Bruno Schoch y Hans-Joachim Spanger (2020): The Nagorno-Karabakh deadlock, Wiesbaden, Springer.

Secretaría General de Naciones Unidas, 'Statement attributable to the Spokesperson for the Secretary-General - on Nagorno-Karabakh', 4 December 2020, en https://www.un.org/sg/en/content/sg/statement/2020-12-04/statement-attributable-thespokesperson-for-the-secretary-general-nagorno-karabakh

Setién, Sara, 'Conflicto de Nagorno Karabaj 2020: ¿nos encontramos ante la solución definitiva?', Instituto Español de Estudios Estratégicos, IEEE Documentos de Opinión 54/2020, 1 de diciembre de 2020.

Shahnazarian, Nona, 'A Good Soldier and a Good Mother: New Conditions and New Roles in the Nagorno-Karabakh War', The Journal of Power Institutions in Post-Soviet Studies (2017), en https://journals.openedition.org/pipss/4241 
Tskhvariashvili, Sophio, 'Gender Implications of the Nagorno-Karabakh Conflict: Alternative Feminist Perspectives', Tesis de Máster, Ivane Javakhishvili Tbilisi State University, 2018.

Van Severen, Sebastiaan 'Lavrov's Lament: A Russian take on the rules-based global order', en EJIL: Talk! Blog of the European Journal of International Law, July 2021, en https://www.ejiltalk.org/lavrovs-lament-a-russian-take-on-the-rules-based-global-order/ 